\title{
Empirical Research on the Influence Factors of E-commerce Development in China
}

\author{
Xiaohong Wang* and Laiyu Liu
}

Management College, Beijing Union University, Beijing, 100101, China

\begin{abstract}
Due to multiple factors affecting e-commerce development, in the environment of network growth, specifically the dramatic increase of mobile Internet users, how to grasp the key influence factors and enhance the healthy development of e-commerce in China? This paper discusses the influence factors of e-commerce development from the perspectives of information infrastructure construction, economic level, educational level, urbanization level, technology level, living standards, human capital level and price index. Based on the time series data from year 2000 to 2012, this paper proposes the influence factor model of e-commerce development by partial least square regression, and analyzes the influence factors using correlation coefficient and variable importance in projection. The research findings show that the most critical factors affecting e-commerce development in China are mobile phone penetration, per capita disposable income, number of computers per hundred households and urbanization rate; and real GDP per capita, knowledge index and Internet penetration also have a certain impact on e-commerce development. This paper reveals the key factors affecting e-commerce development and puts forward some reasonable suggestions in the aspects of information infrastructure construction, mobile e-commerce development, people's living standards and cultural quality.
\end{abstract}

Keywords: E-commerce, influence factor, partial least square method, principal component, variable importance in projection.

\section{INTRODUCTION}

The influence of e-commerce on economy has gradually been obvious and deepened with the global electronic tendency. E-commerce is becoming more and more popular in the world because of its convenience and without time and space limitation. E-commerce is a new economic activity and has become an important part of strategic emerging industries and modern distribution ways in China. With the rapid development of Internet technology, e-commerce with the fusion of real economic activity has become an important driving force to adjust economic structure. During the 11th five-year plan, China's e-commerce development has maintained a good momentum, with the turnover increased by nearly 2.5 times. The concept of triple-networks convergence and the development of Internet and cloud computing technology have produced important influence on e-commerce development, and expanded the space of e-commerce development. With the deepening development of industrialization and informatization, urbanization, marketization and internationalization, e-commerce will enter a period of rapid development in China. According to the 12th five-year development plan of e-commerce, China's e-commerce turnover will quadruple and exceed 18 trillion yuan by 2015 [1]. Under this background, the empirical research on influence factors of e-commerce development in China has strong practical significance.
Now, many scholars conduct the research on e-commerce development from different perspectives. Most research perspectives could be summarized as follows:

One of the perspectives is based on the roles and influence factors of e-commerce. Martin Brooks and Zaki Wahhaj used multi-regional econometric model to estimate the impact of B2B e-commerce on macroeconomic of USA, Japan, Germany, Britain and France [2]. Yang Jianzheng, zhou Tao and Li Qingzi employed Douglas function method to probe into the relationship between e-commerce and economic growth, with the results indicating that e-commerce had the obvious role in promoting economic growth, and the lower e-commerce safety index was one of the main reasons hindering e-commerce development [3]. Li Jianchuan and Zhang Xianfeng thought the main factors affecting ecommerce development in western China were e-commerce consciousness, logistics system and network credit degree, business management, communications infrastructure, ecommerce talents and legal issues [4]. You Guiqiao pointed out the key factors affecting the growth of e-commerce network were social factors, technical factors, management factors, economic factors and competitive factors [5].

The second perspective is based on the development level or e-readiness measurement of e-commerce. Many organizations, enterprises, universities had carried out the relevant research from different angles. Asia-Pacific Economic Cooperation Organization (APEC) launched the index system of e-readiness evaluation guidance based on e-readiness concept [6]. The e-readiness research group of USA Massachusetts Institute of Technology established an evaluation 
system which included access, ability and opportunity [7]. Cisco put forward an evaluation method (Net-ready Evaluation) for enterprise e-commerce level which mainly included leadership, management, ability and technology four categories with 57 specific indexes [8]. China Internet Research and Development Center built the total index system of ecommerce in 2001 [9]. Huang Jinghua, Huang He and Zhao Chunjun established the evaluate index in view of enterprise e-commerce, including enterprise external environment, enterprise internal demand, information technology and enterprise culture three categories with 67 specific indexes [10]. Liu Yue and Wang Wenqing set up the index model of regional e-commerce development on the basis of regional economics, including e-commerce trade index, information index, e-commerce human capital index three categories with 11 specific indexes [11]. Huang Jianqing, Shui Miao and Chai Wenyi used e-readiness model for trading system to evaluate the development level of e-readiness of 7 countries, from the aspects of infrastructure, social environment, market conditions, consumers and enterprises [12].

The third perspective is based on the adoption of ecommerce. Taking the B2B e-commerce market as an example, Zhao Jing, Wang Shan and Huang Wilfred said the key factors affecting e-commerce adoption in China was due to the lack of infrastructure, technology and services [13]. Tan Xiaolin and Zhou Jianhua classified the factors affecting ecommerce adoption as several aspects of economic structure, infrastructure, population structure, culture, system, market size and government policy [14]. Xie Wei and Li peixin founded the comprehensive factors framework for enterprise e-commerce adoption, and concluded that the three factors affecting enterprise e-commerce adoption decisions are external environment, enterprise itself and e-commerce technology [15].

The previous research achievements have reference value for the future research. There are many factors affecting ecommerce development, which not only relate to the development of computer technology and Internet technology, but also relate to the national policies, legal system, political environment, consumer psychology and habit, business philosophy, social and cultural background. Compared with the developed countries, China's e-commerce market has its own uniqueness. The development of mobile Internet users is quicker than the development of Internet users, and this factor is rarely considered in the current study. From the aspects of research methods, there are some problems to affect the validity of research results, such as incomplete index, short data sequence, and serious multicollinearity of influence factors. Considering the particular advantages of partial least square (PLS) regression in analyzing multivariate data with few observations, analyzing the factors affecting ecommerce development, and filtering the factors by variable importance in projection, this paper proposes the influence factor model of e-commerce development by PLS regression. Firstly, it builds the research model using the time series data from year 2000 to 2012 on the basis of comprehensive consideration of various factors, and then analyzes the main influence factors using correlation coefficient and the index of variable importance in projection. We hope this research can promote e-commerce development in China.
This paper explains how to perform the empirical analysis in detail. First of all, it describes the research background and the literature review; secondly, it explains the principle of research model and how to build the regression model; thirdly, it presents how to collect and process data, and analyzes the survey results; fourthly, it provides some suggestions to prompt and improve e-commerce development according to the analysis results; at the end of the full text, it comes up with the foresight and future work.

\section{MODEL AND METHODOLOGY}

\subsection{Model Building}

Based on reviewing the related literature and combining with the actual situation of e-commerce development in China, this paper takes the development level of e-commerce as dependent variable and adopts the proportion of ecommerce turnover accounted for GDP as measure index, which can reflect the position and role of e-commerce in the national economy. The factors affecting e-commerce development can be divided into 8 categories, that is, information infrastructure construction, economic level, educational level, urbanization level, technology level, living standards, human capital level and price index. This paper selects 12 indexes as independent variables in advance, as shown in Table 1, based on literatures of e-commerce research.

The specific role of these 12 indexes is as follows:

- Internet penetration: It reflects the population proportion of a country or area using the Internet. With the rise of Internet penetration in China, Internet has a considerable impact on people's lifestyles, communication and so on.

- Number of computers per hundred households: It refers to the average number of computer ownership per hundred households.

- Number of websites per ten thousand people: It is equal to the total number of websites divided by total population, and then multiplied by 10000 .

- Mobile phone penetration: It refers to the average number of mobile phones per one hundred people.

- Real GDP per capita: It reflects the situation of a country's economic development.

- Knowledge index: It is equal to the adult literacy rate $x$ $2 / 3+$ the average years of education $\times 1 / 3$. It is used to measure the education development level of country.

- Urbanization rate: It is used to reflect the process and degree of population aggregating to the city.

- R\&D spending as a share of GDP: It is used to measure the size of national science and technology activities, the level of investment in science and technology, and the ability of science and technology innovation.

- Number of patent applications per ten thousand people: It is equal to the patent grant divided by total population, and then multiplied by 10000 .

- Per capita disposable income: It reflects the changes of people's living standards in a country. 
Table 1. The selection of dependent and independent variables.

\begin{tabular}{|c|c|}
\hline Development level of e-commerce & Proportion of e-commerce turnover accounted for GDP $(Y)$ \\
\hline Information infrastructure construction & Number of computers per hundred households $\left(X_{2}\right)$ \\
\hline Economic level & Real GDP per capita $\left(X_{5}\right)$ \\
\hline Educational level & Knowledge index $\left(X_{6}\right)$ \\
\hline Urbanization level & Urbanization rate $\left(X_{7}\right)$ \\
\hline Human capital level & Proportion-based employment of information industry $\left(X_{11}\right)$ \\
\hline Price index & Communication price index $\left(X_{12}\right)$ \\
\hline
\end{tabular}

- Proportion-based employment of information industry: It is equal to the number of information industry employees divided by total number of employees.

- Communication price index: It reflects the change direction, trend and degree of communication goods price level in the different period.

Based on literatures of e-commerce research, and principles of comprehensiveness, objectivity and comparability, and data availability, this paper selects the mean indexes or proportion indexes to build data model. Because linear model can specify the relationship between dependent variable $Y$, and a set of independent variables $X$, this paper builds the influence factor model of e-commerce development, as shown in equation (1).

$$
\begin{aligned}
Y= & \beta_{0}+\beta_{1} X_{1}+\beta_{2} X_{2}+\ldots \beta_{i} X_{i} \\
& i=1,2, \ldots, 13 \text { (from year } 2000 \text { to } 2012 \text { ) }
\end{aligned}
$$

In this equation, $\beta_{0}$ is the regression coefficient for intercept, and the $\beta_{i}$ values are the regression coefficient of independent variables and the $i$ represents the number of independent variables. Using this model, it is possible to investigate which combinations of factors have the greatest influence on e-commerce development.

\subsection{Methodology}

PLS was introduced by the Swedish statistician Herman Wold, who then developed it with his son, Svante Wold. Although the original applications were in the social sciences, PLS regression is today most widely used in chemometrics and related areas. It is also used in bioinformatics, sensometrics, neuroscience and anthropology. PLS is a multivariate statistical data analysis method and is particularly useful when we need to predict a set of dependent variables from a large set of independent variables. PLS regres- sion generalizes and combines the features from principal component analysis (PCA), canonical correlation analysis (CCA) and multivariable linear regression analysis.

PLS is a linear system identification method that determines a latent space with orthogonal principle factors to approximate the original input-output space. PLS rearranges argument information using the method of information decomposition and can effectively extract the principal components, which can establish forecast model in severe multiple correlation between variables without considering principal components and dependent variables related limitations; it includes all original independent variables in the final model to avoid the disadvantages of stepwise regression analysis method and maximize data information; and there is no special requirements to sample number. The principle of PLS model is as follows:

Assuming that the set of $q$ dependent variables is $Y, Y$ $=\left\{Y_{1}, Y_{2}, \ldots, Y_{q}\right\}$ and the set of $p$ independent variables is $X$, $X=\left\{X_{1}, X_{2}, \ldots, X_{p}\right\}$. To study the statistical relationship between dependent variables and independent variables, $\mathrm{n}$ samples are observed and the data of independent variables and dependent variables $X=\left\{X_{1}, X_{2}, \ldots, X_{p}\right\}_{n \times p}$ and $Y=\left\{Y_{1}\right.$, $\left.Y_{2}, \ldots, Y_{q}\right\}_{n \times q}$ are constituted. PLS regression extracts principal components $t_{1}$ and $u_{1}$ from $X$ and $Y$ respectively. The $t_{1}$ and $u_{1}$ are respectively the linear combination of $X_{1}, X_{2}, \ldots$, $X_{p}$ and $Y_{1}, Y_{2}, \ldots, Y_{q}$. The principal components $t_{1}$ and $u_{1}$ carry the original variation information as much as possible and the relevance of $t_{1}$ and $u_{1}$ is supposed to be the biggest. After extracting the first principal component $t_{1}$ and $u_{1}$, PLS implements $X$ regression of $t_{1}$ and $Y$ regression of $t_{1}$ respectively. The algorithm will be terminated if the regression equation reaches a satisfactory accuracy, while it continues to extract the second principal component until reaching the 


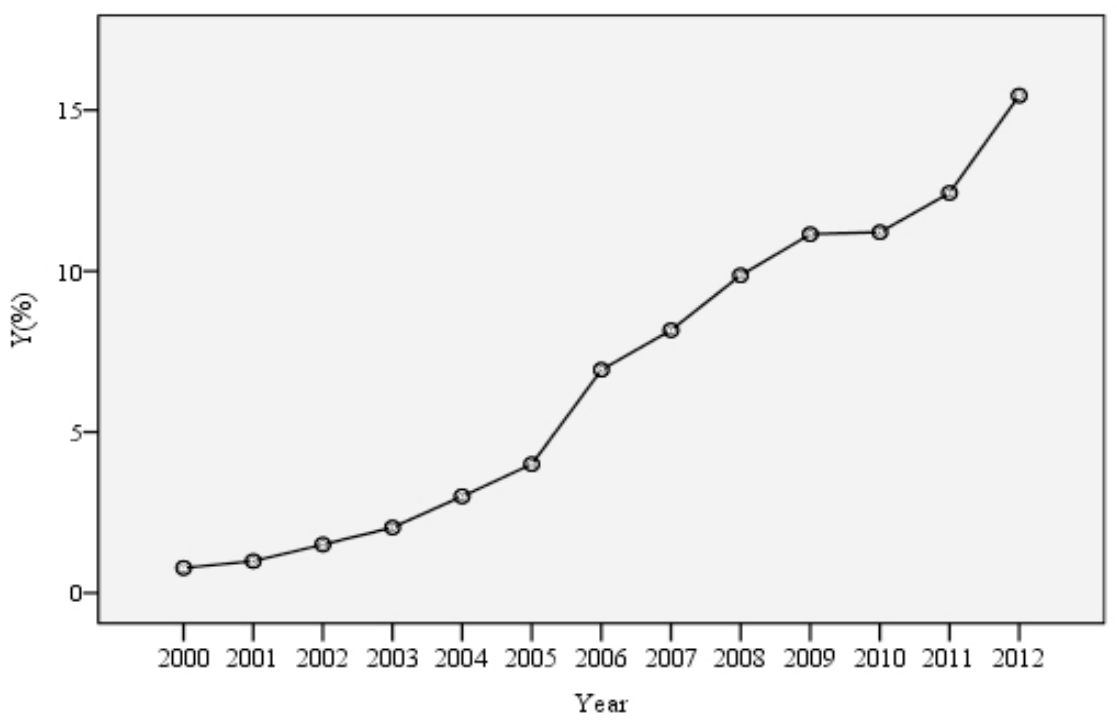

Fig. (1). Proportion of e-commerce turnover accounted for GDP from year 2000 to 2012.

satisfactory accuracy. If there are $m$ principal components $t_{1}$, $t_{2}, \ldots, t_{m}$ extracting from $X$, PLS will build the regression equation of $Y_{k}(k=1,2, \ldots, q)$ and $t_{1}, t_{2}, \ldots, t_{m}$, and then represent as the regression equation of $Y_{k}(k=1,2, \ldots, q)$ and original independent variables $X_{1}, X_{2}, \ldots, X_{p}[16]$.

In this paper, we explore the model structure based on PLS. The basic steps of PLS regression analysis are as follows: It firstly determines whether there is a relationship between dependent variable $(Y)$ and independent variables $(X)$, and this is the premise for establishing model; then it makes multicollinearity diagnosis for independent variables $(X)$ using variance inflation factor (VIF), and this is the precondition to determine whether the model is suitable for PLS; finally, it extracts the principal components and estimates the regression coefficient.

\section{DEMONSTRATION STUDY}

\section{1. Data Collection}

In order to validate the data model, this paper uses the time series data from year 2000 to 2012 . The basic data used in this research are those derived from China information almanac, Chinese statistical yearbook, China intellectual property statistical yearbook and China's e-commerce report. Among them, the data of e-commerce turnover accounted for GDP are available from China's e-commerce report, as shown in Fig. (1); the data of Internet penetration, number of computers per hundred households, mobile phone penetration, number of websites, knowledge index and R\&D spending as a share of GDP are available from China information almanac; the data of patent applications are available from China intellectual property statistical yearbook; the data of real GDP per capita, per capita disposable income, urbanization rate, number of information industry employees, total number of employees and communication price index are available from Chinese statistical yearbook.
Due to different units of each index, this paper has carried on the dimensionless processing using SPSS before building model.

\subsection{Data Processing}

Analyze the correlation between variables. Person correlation coefficient between variables is calculated by SPSS, as shown in Table 2. Person correlation coefficient is widely used to measure the degree of linear correlation between two variables, giving a value between 1 and -1 , where 1 is total positive correlation, 0 is no correlation, and -1 is total negative correlation. Person correlation coefficient with its absolute value above 0.8 usually states there is a stronger linear relationship between two variables. As Table 2 shows that dependent variable $Y$ and independent variables $X_{1} \sim X_{11}$ have high correlation at the $1 \%$ probability level (the value of person correlation coefficient are greater than 0.9 ), except independent variable $X_{12}$. This reveals that variables selection and model building are correct. Most of independent variables $X$ have high correlation with each other at the $1 \%$ probability level (the value of person correlation coefficient are greater than 0.8 ).

Variance inflation factor (VIF) is used to further test the multicollinearity between independent variables $X$. The presence of multicollinearity within the set of independent variables can cause a number of problems in understanding the significance of individual independent variables in the regression model. VIF can be used to identify multicollinearity so that the model can be adjusted. When significant multicollinearity exists, the value of VIF will be very large for the independent variables involved. Usually, the VIF above 10 illustrates there is the more serious multicollinearity between two independent variables. The VIF of independent variables $X_{1} \sim X_{12}$ are 799.267, 6109.405, 8.410, 16381.773, 2454.785, 330.857, 1933.498, 152.084, 173.006, 18835.708, 797.313 and 16.860 respectively. Thus, PLS is used for data regression analysis in this paper. 
Table 2. The person correlation coefficients of variables.

\begin{tabular}{|c|c|c|c|c|c|c|c|c|c|c|c|c|c|}
\hline & $\boldsymbol{Y}$ & $X_{1}$ & $X_{2}$ & $X_{3}$ & $X_{4}$ & $X_{5}$ & $X_{6}$ & $X_{7}$ & $X_{8}$ & $X_{9}$ & $X_{10}$ & $X_{11}$ & $X_{12}$ \\
\hline $\boldsymbol{Y}$ & 1 & $0.969^{* *}$ & $0.982^{* *}$ & $0.902^{* *}$ & $0.983^{* *}$ & $0.980^{* *}$ & $0.970^{* *}$ & $0.981^{* *}$ & $0.968^{* *}$ & $0.961^{* *}$ & $0.984^{* *}$ & $0.936^{* *}$ & 0.481 \\
\hline$X_{1}$ & $0.969^{* *}$ & 1 & $0.962^{* *}$ & $0.867^{* *}$ & $0.986^{* *}$ & $0.993^{* *}$ & $0.987^{* *}$ & $0.962^{* *}$ & $0.975^{* *}$ & $0.984^{* *}$ & $0.991^{* *}$ & $0.914^{* *}$ & 0.513 \\
\hline$X_{2}$ & $0.982^{* *}$ & $0.962^{* *}$ & 1 & $0.864^{* *}$ & $0.992^{* *}$ & $0.977^{* *}$ & $0.976^{* *}$ & $0.999^{* *}$ & $0.974^{* *}$ & $0.955^{* *}$ & $0.981^{* *}$ & $0.977^{* *}$ & 0.552 \\
\hline$X_{3}$ & $0.902^{* *}$ & $0.867^{* *}$ & $0.864^{* *}$ & 1 & $0.856^{* *}$ & $0.851^{* *}$ & $0.848^{* *}$ & $0.864^{* *}$ & $0.833^{* *}$ & $0.811^{* *}$ & $0.859^{* *}$ & $0.849^{* *}$ & 0.256 \\
\hline$X_{4}$ & $0.983^{* *}$ & $0.986^{* *}$ & $0.992^{* *}$ & $0.856^{* *}$ & 1 & $0.994^{* *}$ & $0.991^{* *}$ & $0.991^{* *}$ & $0.986^{* *}$ & $0.983^{* *}$ & $0.997^{* *}$ & $0.955^{* *}$ & $0.567^{*}$ \\
\hline$X_{5}$ & $0.980^{* *}$ & $0.993^{* *}$ & $0.977^{* *}$ & $0.851^{* *}$ & $0.994^{* *}$ & 1 & $0.987^{* *}$ & $0.974^{* *}$ & $0.978^{* *}$ & $0.992^{* *}$ & $0.999^{* *}$ & $0.921^{* *}$ & 0.532 \\
\hline$X_{6}$ & $0.970^{* *}$ & $0.987^{* *}$ & $0.976^{* *}$ & $0.848^{* *}$ & $0.991^{* *}$ & $0.987^{* *}$ & 1 & $0.979^{* *}$ & $0.978^{* *}$ & $0.975^{* *}$ & $0.988^{* *}$ & $0.948^{* *}$ & $0.582^{*}$ \\
\hline$X_{7}$ & $0.981^{* *}$ & $0.962^{* *}$ & $0.999^{* *}$ & $0.864^{* *}$ & $0.991^{* *}$ & $0.974^{* *}$ & $0.979^{* *}$ & 1 & $0.974^{* *}$ & $0.951^{* *}$ & $0.979^{* *}$ & $0.982^{* *}$ & $0.571^{*}$ \\
\hline$X_{8}$ & $0.968^{* *}$ & $0.975^{* *}$ & $0.974^{* *}$ & $0.833^{* *}$ & $0.986^{* *}$ & $0.978^{* *}$ & $0.978^{* *}$ & $0.974^{* *}$ & 1 & $0.972^{* *}$ & $0.981^{* *}$ & $0.937^{* *}$ & $0.605^{*}$ \\
\hline$X_{9}$ & $0.961^{* *}$ & $0.984^{* *}$ & $0.955^{* *}$ & $0.811^{* *}$ & $0.983^{* *}$ & $0.992^{* *}$ & $0.975^{* *}$ & $0.951^{* *}$ & $0.972^{* *}$ & 1 & $0.992^{* *}$ & $0.888^{* *}$ & $0.555^{*}$ \\
\hline$X_{10}$ & $0.984^{* *}$ & $0.991^{* *}$ & $0.981^{* *}$ & $0.859^{* *}$ & $0.997^{* *}$ & $0.999^{* *}$ & $0.988^{* *}$ & $0.979^{* *}$ & $0.981^{* *}$ & $0.992^{* *}$ & 1 & $0.930^{* *}$ & 0.542 \\
\hline$X_{11}$ & $0.936^{* *}$ & $0.914^{* *}$ & $0.977^{* *}$ & $0.849^{* *}$ & $0.955^{* *}$ & $0.921^{* *}$ & $0.948^{* *}$ & $0.982^{* *}$ & $0.937^{* *}$ & $0.888^{* *}$ & $0.930^{* *}$ & 1 & $0.593^{*}$ \\
\hline$X_{12}$ & 0.481 & 0.513 & 0.552 & 0.256 & $0.567^{*}$ & 0.532 & $0.582^{*}$ & $0.571^{*}$ & $0.605^{*}$ & $0.555^{*}$ & 0.542 & $0.593^{*}$ & 1 \\
\hline
\end{tabular}

$* * . \alpha$ is the $1 \%$ probability level; $*$. $\alpha$ is the $5 \%$ probability level.

Table 3. Proportion of variance explained.

\begin{tabular}{|c|c|c|c|c|c|}
\hline \multirow{2}{*}{ Latent Factors } & \multicolumn{5}{|c|}{ Statistics } \\
\cline { 2 - 7 } & $\boldsymbol{X}$ Variance & Cumulative $\boldsymbol{X}$ Variance & $\boldsymbol{Y}$ Variance & Cumulative $\boldsymbol{Y}$ Variance (R $^{2}$ ) & ${\text { Adjusted } \mathbf{R}^{2}}^{2}$ \\
\hline \hline 1 & 0.903 & 0.903 & 0.968 & 0.968 & 0.981 \\
\hline 2 & 0.066 & 0.969 & 0.013 & 0.988 & 0.977 \\
\hline 3 & 0.007 & 0.976 & 0.007 & 0.991 & 0.984 \\
\hline 4 & 0.012 & 0.989 & 0.003 & 0.997 & 0.995 \\
\hline 5
\end{tabular}

Determine the principal component. The number of principal components is less than or equal to the number of original variables. This transformation is defined in such a way that the first principal component has the largest possible variance, and each succeeding component in turn has the highest variance possible under the constraint that it is orthogonal to the preceding components. It is very important that the principal components are independent if the data set is jointly normally distributed. Through testing the validity of multiple component of regression equation, it is found that there is only one principal component meeting validity conditions at the $1 \%$ probability level, namely its characteristic value is greater than 1 . Therefore, one PLS component serving as principal component is used for regression model analysis, the proportion of cumulative $X$ variance is $90.3 \%$, the proportion of cumulative $Y$ variance $\left(\mathrm{R}^{2}\right)$ is $96.8 \%$ and adjusted $\mathrm{R}^{2}$ is $96.5 \%$, as shown in Table 3 .

Build model. Based on the principal component, standardization coefficient of each variable is obtained by PLS, as shown in Table 4 . The symbol of standardized coefficient usually signify the direction of independent variables affecting dependent variable, and its size is only roughly to identify the degree of independent variables affecting dependent variable, without taking the contribution of independent variables to principal components.

Calculate the variable importance in projection (VIP). Because VIP considers the contribution of independent variables to principal components and the ability of principal components to explain dependent variable, the relative importance of each independent variable on dependent variable can be accurately judged by VIP. VIP is an indication of the importance of independent variables in predicting the $Y$ space. The VIP of each independent variable is shown in Table 4, its size is $X_{4}=X_{10}>X_{2}>X_{7}>X_{5}>X_{6}>X_{1}=X_{8}>X_{9}>X_{11}>$ $X_{3}>X_{12}$ in turn. Depending on the principle, that is $\operatorname{VIP}_{i}>1$, independent variables $X_{1}, X_{2}, X_{4}, X_{5}, X_{6}, X_{7}, X_{8}, X_{9}, X_{10}$ and $X_{11}$ can play an important role in explaining dependent variable $\mathrm{Y}$, in which $X_{4}$ and $X_{10}$ have the most important role. 
Table 4. The coefficient, standardization coefficient and VIP of variables.

\begin{tabular}{|c|c|c|c|}
\hline Constant & 0 & - & - \\
\hline$X_{2}$ & 0.091 & 0.301 & 1.051 \\
\hline$X_{5}$ & 0.090 & 0.301 & 1.048 \\
\hline$X_{6}$ & 0.090 & 0.302 & 1.038 \\
\hline $\boldsymbol{X}_{7}$ & 0.091 & 0.301 & 1.049 \\
\hline$X_{11}$ & 0.086 & 0.292 & 1.002 \\
\hline$X_{12}$ & 0.044 & 0.176 & 0.515 \\
\hline
\end{tabular}

Based on the previous survey, it is shown that the model is through the validity of PLS regression test, and this model overcomes the interference of multiple linear and is significant at the $1 \%$ probability level. The proportion of cumulative $X$ variance is above $90 \%$ and $\mathrm{AR}^{2}$ is above $96 \%$, which indicates that dependent variables of this model are more effective with less interference.

\section{DISCUSSION AND SUGGESTION}

\subsection{Discussion}

The research results of time series data from year 2000 to 2012 indicate that the factors affecting e-commerce development in China are mobile phone penetration, per capita disposable income, number of computers per hundred households, urbanization rate, real GDP per capita, knowledge index, Internet penetration, R\&D spending as a share of GDP, number of patent applications per ten thousand people and proportion-based employment of information industry according to its contribution degree. The empirical analysis demonstrates that the most critical factors are mobile phone penetration, per capita disposable income, number of computers per hundred households, and urbanization rate; and real GDP per capita, knowledge index and Internet penetration are the more important factors. The impact of number of websites per ten thousand people and communication price index on e-commerce development in China are less significant.

\subsection{Suggestions}

Based on the analysis results, some reasonable suggestions on e-commerce development are put forward from the following aspects.

Further increase the informatization infrastructure construction. Mobile phone penetration and number of computers per hundred households are the key driving factors pro- moting e-commerce development in China. The development of successful e-commerce initiatives requires creation of an appropriate infrastructure that will support the required functionality. Good e-commerce infrastructure is the foundation of e-commerce development, which must be able to support transaction process with continuous development and increasingly complex. To promote the rapid development of e-commerce, it is very necessary to increase investment in network infrastructure to make it better.

Pay attention to the development of mobile e-commerce. According to the statistical report on Internet development in China, by the end of December 2013, the number of Internet users in China had reached 618 million and the Internet penetration was $45.8 \%$, of which the number of mobile Internet users had reached 500 million and kept growing. The continuous growth of mobile Internet users facilitated the development of various applications of mobile phone terminal and was a highlight of China's Internet development in 2013 [17]. The popularity of mobile phones and the improvement of urbanization level will effectively promote ecommerce development. With the rapid development of $3 \mathrm{G}$ and $4 \mathrm{G}$ networks, the development of mobile e-commerce has entered an unprecedented era.

Improve the national economy and people's living standards. Per capita disposable income and real GDP per capita are also the important factors driving e-commerce development in China. With the improvement of living standards, people's demand for high quality and differentiated goods is also growing, and this will put forward higher requirements for e-commerce. The enhancement of national economy will promote the overall growth of e-commerce, and in turn the ecommerce development will further promote the rapid development of national economy.

Improve the cultural quality. Now, there are some problems affecting people's enthusiasm engaged in e-commerce, 
such as less standardized domestic market mechanism, immature e-commerce environment, and the lack of credit guarantee system. It is very important to improve national culture quality to create a good consumption environment for e-commerce, especially to further deepen the understanding of e-commerce to make people really realize the convenience of e-commerce, such as price and service advantages.

\section{CONCLUSION}

Due to multiple factors affecting e-commerce development in China, this paper has carried out empirical research on the key factors using PLS model based on the time series data from year 2000 to 2012 . The results show that the most critical factors affecting e-commerce development in China are mobile phone penetration, per capita disposable income, number of computers per hundred households and urbanization rate; and real GDP per capita, knowledge index and Internet penetration also have a certain impact on e-commerce development. In order to promote e-commerce development in China, it is very necessary to further strengthen information infrastructure construction, to attach great importance to mobile e-commerce development, and to pay attention to improvement of people's living standards and cultural quality.

Several valuable conclusions have been drawn from the investigation and study, but there are still some limitations: the research data and the number of samples are both limited. In addition, in the future research, more data and samples will be used to verify the model's value.

\section{CONFLICT OF INTEREST}

The authors confirm that this article content has no conflict of interest.

\section{ACKNOWLEDGEMENTS}

This paper is supported by the Philosophy and Social Science Plan Research Projects: Research on the Networked Growth Performance of the Enterprises at the High-tech Industrial Park (11JGB040), from the Beijing Municipal Government.

We wish to thank Professor Zhang Shiyu and Tao Qiuyan for data analysis, and thank Li Liwei for valuable discussions. We also want to thank my colleagues, Pei Yilei, who helped and guided us to translate this paper into English.

\section{REFERENCES}

[1] The Ministry of Industry and Information Technology, The ECommerce $12^{\text {th }}$ Five-Year Development Plan, March 2012.

[2] M. Brookes, and Z. Wahhaj, The Shocking Economic Effect of B2B, Goldman Sachs\& Co, Global Economics, February 2000.

[3] J. Yang, T. Zhou and Q. Li, "An empirical analysis of electronic commerce on economic growth", World Economy Study, vol. 88, no. 10 , pp. 40-43, October 2011.

[4] J. Li, and X. Zhang, "Main factors and countermeasures influencing the development of e-commerce in the western china", Economic Management in China, vol. 27, no. 2, pp. 84-89, February 2007.

[5] G. You, "Factors influencing the growth of electronic commerce network", China Business \& Trade, vol. 20, no. 25, pp. 169-170, September 2011

[6] Asia-pacific economic cooperation, "APEC E-Commerce Readiness Initiative", http://www.ita.doc.gov/td/industry/otea/ ecommerce/apec.

[7] M. Siegel, F. Haghseta, and S. Donnel, Global E-Readiness Opportunities: New Framework and Tools, Cambridge: The center for ebusiness@MIT, 2002.

[8] J. Wei and Y. Xie, "A review on measure theory of electronic commerce level", Journal of Information, vol. 25, no. 2, pp. 91-93, February 2006

[9] CII Electronic Commerce Index Research and Measurement Research Group, "Research on e-commerce level measurement", Statistical Research, vol. 18, no. 12, pp. 26-30, December 2001.

[10] J. Huang, H. He, and C. Zhao, "The Indicator for Enterprises EReadiness Evaluation and Its Application", Journal of Tsinghua University (Philosophy and Social Science), vol. 19, no. 3, pp. 6369, 92, March 2004.

[11] Y. Liu and W. Wang, "The reconstruction and empirical analysis of regional e-commerce development index", Science of Science and Management of S. \& T., vol. 30, no. 7, pp. 144-147, July 2009.

[12] H. Jianqing, S. Miao, and C. Wenyi, "The model and empirical study of the electronic commerce development level of readiness", Statistics and Decision, vol. 336, no. 18, pp. 100-103, September 2012.

[13] J. Zhao, S. Wang, and W. V. Huang, "A study of B2B e-market in china: E-commerce process perspective", Information \& Management, vol. 45, no. 4, pp. 242-248, April 2008.

[14] X. Tan, and J. Zhou, "Key factors that influence the adoption of e-commerce", China Soft Science, vol. 28, no. 1, pp. 182-192, January 2013.

[15] W. Xie, and P. Li, "Key factors influencing firms' adoption of e-commerce", Economic Management, vol. 34, no. 2, pp. 150-158, February 2012

[16] H. Wang, Partial Least Squares Regression Method and Its Application, In:Beijing: National Defense Industry Press, April 1999.

[17] China Internet network information center, The $33^{\text {rd }}$ Statistical Report on Internet Development in China, January 2014.

\footnotetext{
Received: September 22, 2014

Revised: November 30, 2014

Accepted: December 02, 2014

(C) Wang and Liu; Licensee Bentham Open.

This is an open access article licensed under the terms of the Creative Commons Attribution Non-Commercial License (http://creativecommons.org/licenses/by-nc/3.0/) which permits unrestricted, non-commercial use, distribution and reproduction in any medium, provided the work is properly cited.
} 